\title{
Evaluation of a Study Instrument Assessing Community Pharmacies and Services Provided: Consumers' Perception
}

\author{
Clement Liew Tze How ${ }^{1}$, Sam Aaseer Thamby ${ }^{2,}$, Ng Yen Ping $^{2}$ \\ ${ }^{1}$ Faculty of Pharmacy, AIMST University, MALAYSIA. \\ ${ }^{2}$ Clinical Pharmacy and Pharmacy Practice Unit, Faculty of Pharmacy, AIMST University, MALAYSIA.
}

\begin{abstract}
Objectives: The objective of this research is to assess and compare the degree of correlation and agreement between the three study instruments utilized in evaluation of community pharmacies and services provided based on consumer's perception. Methods: A cross sectional observational study on a convenient random sample of 100 respondents from Sungai Petain, Kedah, Malaysia was conducted by using pretested and validated questionnaires to evaluate the community pharmacies and the services provided by them based on consumers' perception. The data retrieved from the three set of questionnaires were coded based upon various variables into Microsoft Excel 2010 and were then exported to the IBM SPSS Version 22.0 to be analyzed. The Chi-square test and Kappa concordant test were used to test the statistical significance and the degree of agreement between the three domains utilized respectively. Results: Totally 41 males and 59 females participated in this study. The result analyses clearly showed that despite the similarities, there did not exist any degree of agreement between the three instruments (by Spearman's correlational test, Kappa concordant test). Fortifying this observation was the score grades assessment, wherein all the three sets, especially the PSQ-3 and PSQ-18 gave extremely varied scores and corresponding score
\end{abstract}

grades. Conclusion: The principal investigator thus concluded (based on the study parameters set) that though the three instruments measure and analyze the same concept, each of them are distinctive by themselves. This conclusion implies that for future research involving patients' satisfaction with community pharmacy services, the aspiring researcher must exercise extreme caution while combining these three questionnaires to create an adapted version of a new questionnaire to assess the same.

Key words: Patient Satisfaction Questionnaire, Community Pharmacy Patient Questionnaire, Community Pharmacy, Community Pharmacist, Consumer's Perception, Kappa Concordant value.

Correspondence

Dr. Sam Aaseer Thamby,

Senior Lecturer, Faculty of Pharmacy, AIMST University, Bedong-08100, Kedah, MALAYSIA.

Phone: +600164266240

Email:sam_aseer@aimst.edu.my

DOI: 10.5530/jyp.2019.11.79

\section{INTRODUCTION}

Pharmacy is a recognized part of medical practice dating as far back as Sumerian times, around 2,000 to 1,500 BC, from which cuneiform tablets have been preserved recording prescribed medications. ${ }^{1}$ Community pharmacists are the health professionals most accessible to the public. They supply medicines in accordance with a prescription or, when legally permitted, sell them without a prescription. In addition to ensuring an accurate supply of appropriate products, their professional activities also cover counselling of patients at the time of dispensing of prescription and non-prescription drugs, drug information to health professionals, patients and the general public and participation in health-promotion programme. The community pharmacy industry is an increasingly competitive sector, where independent pharmacies must compete with national and multinational chains. In this competitive era, each pharmacy seeks to differentiate themselves from others and earn customer trust. Attitudes of pharmacists and pharmacy staff, facilities, value-addedservices and environmental factors play an important part towards perception of a consumer towards a pharmacy. Patient-centric care is one of the key determinants of consumers' perception towards community pharmacists. ${ }^{2}$ Consumer (patients') satisfaction is an integral component of health care's quality. ${ }^{3}$ Cleary and McNeil defined patient satisfaction as "the health care recipient's reaction to salient aspects of his / her service experience". The pharmacists' professional value must be considered in the context of consumers' perception. Unless patients appropriately understand the pharmacist's professional roles with respect to direct patient care, the successful implementation of the pharmaceutical care framework in pharmacies cannot be attained. ${ }^{5}$

In Malaysia, the community pharmacy is usually referred to as a setting where pharmacists practice their profession in the setting of a retail storefront with a dispensary area to store and dispense medications. Community pharmacies aim to provide better healthcare to the public and improve their quality of life (QoL) by rationally providing medication(s) with appropriate drug-related information and counselling, reducing medication errors (MEs). The ultimate goal is to ensure the optimal therapy of drugs by contributing to the preparation, supply, administration and control of medications; and providing counselling to patients; and conducting public health campaigns from the pharmacists' perspective. Community pharmacies are divided into: chain and independent pharmacies; with their own set of operational, management, execution and business strategies. Large-chain pharmacies are usually managed by a large corporation with a strong management team. Every chain pharmacies will have a Standard Operation Procedure (SOP), with standardized prices for products. Independent pharmacies are small scale businesses owned either by a private owner or the pharmacists themselves and operated by pharmacists. The main challenge faced by independent pharmacies is the highly competitive business environment. ${ }^{6}$

In March 2014, the concept of 'Seven Star Pharmacist' introduced by World Health Organization (WHO) and International Pharmaceutical

This is an open access article distributed under the terms of the Creative Commons Attribution-NonCommercial-ShareAlike 4.0 License, which allows others to remix, tweak, and build upon the work non-commercially, as long as the author is credited and the new creations are licensed under the identical terms. 
Federation (IPF) mandated every pharmacist to possess or acquire the following attributes in order to fulfill their responsibilities: Care Giver, Communicator, Decision Maker, Teacher, Lifelong learner, Leader and Manager. ${ }^{78}$ The current survey was conducted to evaluate the community pharmacies including the services provided by the community pharmacists in the context of the above attributes. The main subject of this research is concerning on evaluation of community pharmacies and its services based upon the perception of consumers. The aim of this research was to study the perception of customers (patients) towards community pharmacy and services and their satisfaction with the same, by utilizing three versions of study instruments analyzing the aforementioned phenomena (i.e., patients' satisfaction with community pharmacy services). Objective of this research is to assess and compare the degree of correlation and agreement between the three study instruments utilized in evaluation of community pharmacies and services provided based on consumer's perception. To assess and compare the degree of correlation and agreement between the three study instruments utilized in evaluation of community pharmacies and services provided based on consumer's perception.

\section{METHODS}

This concurrent, exploratory research was conducted on randomly selected respondents from a private university and the general public in Kedah state of Malaysia, to evaluate their attitude, knowledge and perception towards community pharmacies and services. The optimal sample size as estimated by the Raosoft software was 100 . The Raosoft sample size calculator was used for the estimation of sample size on account of the convenience sampling technique. ${ }^{9}$ The ethical approval for the conduct of this study was obtained from the AIMST University Human and Animal Ethics Committee (AUHAEC6/FOP/2018/16). The inclusion criteria were Malaysian citizens residing in Sungai Petani district with the ability to comprehend, read and write; whereas, the exclusion criteria were expatriates, Malaysians unable to comprehend, read and write; and respondents who returned incomplete questionnaires.

\section{Study Instrument}

Three self-administered questionnaires: Community Pharmacy Patient Questionnaire, ${ }^{10}$ Patient Satisfaction Questionnaire-3 $3^{11}$ and Patient Satisfaction Questionnaire- $18^{11}$ all three of which have already proven reliability and validity were provided to the respondents. The questionnaires pertained to the consumers' perception(s) towards community pharmacies focusing on the knowledge, communication skills, ability of management, concern, services provided, facilities and location of pharmacy. The respondents were required to answer all the questions by their own without interference from others. The survey questionnaire will be administered within one year of research period. Prior to distributing the study instrument to each respondent, their consent to voluntarily participate in this study was obtained by the Informed Consent Form (ICF). The respondents were allowed to withdraw from the study at any point. The flow of the study is depicted in Figure 1.

The score range in the CPPQ questionnaire for all the section is ' $0-70$ '. The Patient Satisfaction Questionnaire-3 and Patient Satisfaction Questionnaire-18 have 5-scale Likert scoring system. Respondents are offered a choice of five to seven or nine pre-coded responses with the neutral point being neither agree nor disagree. Each of the five responses would have a numerical value which would be used to measure the attitude under investigation. The score ranges for the PSQ-18 and PSQ-3 questionnaires are '18-90' and '30-150' respectively. The scoring grades attributed to each domain and overall, were adopted from Bloom's cut-off points for grading participants' scores, which are $80-100 \%$ correct response (good); $60-79 \%$ (moderate) and $<60 \%$ (poor). This was utilized for the scores pertaining to each domain of knowledge, dietary habits



Figure 1: Workflow of the study.

and physical activities and the overall score pertaining to the questionnaire. The categorizations of the respondents' scores were done according to the Bloom's original cut-off points. ${ }^{12}$

\section{Statistical analysis}

As the data obtained were not normally distributed, non-parametric tests were employed for data analyses, even though the data was numerical. In the CPPQ, chi-square was used for the crosstab of demographic variables against each question. In the PSQ-3, the chi-square was used for significance analysis for each demographic variable and for assessing the significance of the responses to each question. In the PSQ-18 (a derivative of PSQ-3), the chi-square was again used for investigating the response significance to each question. The Kappa Cohen Concordant Test was used to determine the degree of agreement between each of the questionnaires. Besides, the Spearman's correlational test was performed to assess for any correlation between the three sets when the data is converted to categorical. The correlation and degree of agreement between the three domains (or sets) was performed in the current study.

\section{RESULTS}

41 males and 59 females participated in this study. Barring the gender, all the aforementioned variables were deemed statistically significant as revealed by the chi-square test. Table 1 depicts the demographical variables, while Table 2 depicts the medical and medication histories of the study population.

\section{CPPQ Domain}

S1AQ1 assesses the consumer's reason for visiting the pharmacy. Majority (53\%) of the consumers visited the pharmacy to collect a prescription for themselves and only $30 \%$ of the consumers visited the pharmacy to collect prescription for others. S1BQ2a is a question to assess the consumer's perception on the pharmacist's attitude. Majority of the consumers $(42.0 \%)$ think that the pharmacist and other pharmacy staff have a very good attitude. Out of the $42 \%$ majority, $80 \%$ of them are 
Table 1: Frequency Table - demographic variables.

\begin{tabular}{ccccc} 
& & Frequency & Percent & $p$-value \\
\hline \multirow{4}{*}{ Age (in yrs.) } & 16 to 19 & 5 & 5.0 & \\
& 20 to 24 & 71 & 71.0 & \\
& 25 to 34 & 8 & 8.0 & \\
& 35 to 44 & 7 & 7.0 & \\
& 45 to 54 & 5 & 5.0 & \\
Gender & $>55$ & 4 & 4.0 & \\
& Total & 100 & 100.0 & \\
& Male & 41 & 41.0 & \\
& Female & 59 & 59.0 & \\
Marital & Total & 100 & 100.0 & \\
Status & Single & 85 & 85.0 & \\
& Married & 15 & 15.0 & \\
& Total & 100 & 100.0 & \\
Employment & Employed & 19 & 19.0 & \\
Status & Unemployed & 81 & 81.0 & \\
& Total & 100 & 100.0 &
\end{tabular}

( ${ }^{\star}$ Chi-Square test; $\left.\mathrm{p}<.001\right)$

Table 2: Frequency Table - Medical and Medication History.

\begin{tabular}{ccccc}
\hline & & Frequency & Percent & $p$-value \\
\hline \multirow{4}{*}{ Medical } & Nil & 82 & 82.0 & \\
History & Asthma & 5 & 5.0 & \\
& Hypertension & 7 & 7.0 & \\
& Jiabetes Mellitus & 2 & 2.0 & \\
& Eczema & 2 & 2.0 & $<0.001^{*}$ \\
& epilepsy & 1 & 1.0 & \\
& Others & 1 & 1.0 & \\
Tedication & Total & 100 & 100.0 & \\
History & Nil & 86 & 86.0 & \\
& Anti-Diabetic drugs & 2 & 4.0 & \\
& Aronchodilator & 4 & 2.0 & $<0.001^{*}$ \\
& Antihistamines & 1 & 1.0 & \\
& Corticosteroids & 1 & 1.0 & \\
& Epilim & 1 & 1.0 & \\
& Total & 100 & 100.0 &
\end{tabular}

$\left({ }^{\star}\right.$ Chi-Square test; $\left.\mathrm{p}<.001\right)$

between the age of 45 to 54 . S1BQ2b is a question to assess the consumer's perception on the capability of a pharmacist and pharmacy's staff to answer any queries. $44 \%$ of the consumers from all age categories thought that the capability of a pharmacist and pharmacy's staff to answer their queries was fairly good. S1BQ2d is a question to assess the consumer's perception on the services provided by the pharmacist and the pharmacy's staff. Majority of consumers from all age categories (53\%) thought that they received a fairly good service from the pharmacist and the pharmacy's staff. S1BQ2f assesses the consumer's perception on the
Table 3: Degree of correlation between the three domains.

\begin{tabular}{|c|c|c|c|c|c|}
\hline & & & Set 1 & Set 2 & Set 3 \\
\hline \multirow{9}{*}{$\begin{array}{c}\text { Spearman's } \\
\text { rho }\end{array}$} & \multirow{3}{*}{ Set 1} & Correlation Coefficient & 1.000 & . & . \\
\hline & & Sig. (2-tailed) & . & . & . \\
\hline & & $\mathrm{N}$ & 100 & 0 & 0 \\
\hline & \multirow{3}{*}{ Set 2} & Correlation Coefficient & . & 1.000 & . \\
\hline & & Sig. (2-tailed) & . & . & . \\
\hline & & $\mathrm{N}$ & 0 & 100 & 0 \\
\hline & \multirow{3}{*}{ Set 3} & Correlation Coefficient & . & . & 1.000 \\
\hline & & Sig. (2-tailed) & . & . & . \\
\hline & & $\mathrm{N}$ & 0 & 0 & 100 \\
\hline
\end{tabular}

overall attitude, capability and service quality of the pharmacist and the pharmacy's staff. $31 \%$ of consumers were of the opinion that the respective attributes assessed were very good, $57 \%$ of consumers responded fairly good, $9 \%$ reported fairly poor, $3 \%$ responded 'very poor' to the question. S1BQ3a assesses consumers' perception on pharmacist's ability to provide advice on current health problems. Those aged 16 to 19 years though that the pharmacist did fairly well; $4 \%$ of consumers opined that the pharmacist was not able to properly advise them on current health problems. S1BQ3c assesses consumers' perception on pharmacist's ability to handle medication disposing; $42 \%$ felt that the pharmacist did a fairly well job in disposing of medicines that were no longer needed.

\section{PSQ-3 and PSQ-18 Domains}

A five-point Likert scale was used to gauge the respondents' answers to each of the 30 items (questions) in this domain. The responses were 'strongly agree,' 'agree' 'uncertain', 'disagree' and 'strongly disagree'. The scores assigned ranged from ' $1-5$ ' respectively. The statistical significance of the data was assessed by Chi Square Test. The relevant frequency data, average scoring and statistical significance by Chi-square test $(p<0.001)$ for the items in the PSQ-3 domain were statistically significant $(P<0.001)$. An average score of 77.14 ('moderate' grade) for PSQ-3 domain was observed. The responses were for each item were deemed statistically significant, implying that the majority of the respondents felt or perceived the same way (moderately satisfied; average score 77.14) regarding their perception of the community pharmacy they mostly went to and the pharmaceutical care services offered in those establishments.

The PSQ-18 is a modified, concise version of the Patients' Satisfaction Questionnaire-3. The PSQ-18 comprises 18 items. The scoring and the Likert-scaling pattern is the same as the PSQ-3. A score of 45.60 ('poor' grade in terms of satisfaction) for the PSQ-18 domain was observed.

\section{Degree of agreement between the three domains}

All the three domains in the current study (CPPQ, PSQ-3 and PSQ-18) assessed the same concept, which was consumers' satisfaction with the pharmaceutical services. The researchers wanted to establish if there was any degree of agreement between the three domains. For this purpose, the Kappa concordant test was utilized. The average scores for PSQ-3 (77.14) and PSQ-18 (45.60) are different in terms of the grades. Though the PSQ-18 is a derivative of PSQ-3, it is presumed that the mean score and the corresponding score grades would be similar. However, that is not the case in this context. The Kappa concordant test (Table 3) performed for the three pairs (set $1 \mathrm{v}$ set 2 ; set $1 \mathrm{v}$ set 3 ; and set $2 \mathrm{v}$ set 3 ) revealed that there was no degree of agreement between the pairs. This observation was drawn based on the negative scores for measure of agreement between the three sets. ${ }^{13}$ The Kappa concordant values for all three comparisons are negative (Table 3 ), implying that there is no agreement between the sets despite the significance. 


\section{DISCUSSION}

Cross tabulation of the demographic variables against all the questions were performed to determine if any of the variables had any significance in the context of the questions. The explanation for these results pertains to individual variability and the convenience sampling technique. ${ }^{14}$ S1AQ1 assesses the consumer's reason for visiting the pharmacy. $53 \%$ of the consumers visited the pharmacy to collect a prescription for themselves. This finding is subjective in nature. A study by Boardman et al. analyzing how and why consumers utilize the community pharmacists noted that collection of prescription medicines from community pharmacies was reported by almost $60 \%$ of respondents and $40 \%$, who had bought medications in the previous month for themselves or someone else respectively. ${ }^{15}$ This observation can be ascertained to be due to individual variability, convenience sampling technique and location of the study. S1BQ2a assesses the consumer's perception on the pharmacist's attitude. Majority of the consumers $(42.0 \%)$ thought that the pharmacist and other pharmacy staff had a very good attitude while dispensing the medication and during counselling. S1BQ2b assesses the consumer's perception on the capability of a pharmacist and pharmacy's staff to answer any queries. $44 \%$ of the consumers from all age categories thought that the capability of a pharmacist and pharmacy's staff to answer their queries was fairly good. A similar finding was observed in a study by Al-Arifi, assessing the consumers' perception, views and satisfaction with pharmacists' role as health care provider in community pharmacy settings in Saudi Arabia. His conclusion was that though the patients showed better satisfaction, perception and appreciation of the pharmacists' role in the health care team, extra efforts must be put to update and elevate the clinical skills set of community pharmacists. Community pharmacists must reach out to patients, assess their hesitations and proffer solutions for the medication issues faced by each patient or consumer. They should be pro-active in evolving into an effective and indispensable part of the modern healthcare team. They must advise, guide, direct and persuade the patients to comply with the therapeutic regimen. Community pharmacists should update their knowledge base and competencies so as to tender efficient and outstanding pharmaceutical health care. ${ }^{16}$ S1BQ2d assesses the consumer's perception on the services provided by the pharmacist and the pharmacy's staff. Majority of consumers from all age categories (53\%) thought that they received a fairly good service from the pharmacist and the pharmacy's staff but felt that some of the pharmacists ought to update themselves. This finding is similar to a study conducted by Wirth et al. assessing the Maltese consumers' perceptions of the community pharmacists. They found that majority of the consumers were very or fairly satisfied with various pharmacist characteristics like efficiency when dealing with requests, provision of instructions on how to take medications, discretion, professional pharmacist-consumer relationship, explaining how medications work and pharmacist's knowledge and ability to answer drug-related questions. Consumers were least satisfied with the privacy in the pharmacy. Consumers were in favour of the evolution of pharmacist professional services, mainly the community pharmacist liaising with primary and secondary care-based physicians, provision of diagnostic testing and extended opening hours. They concluded that though consumers had a positive overall perception of community pharmacists and of the services offered from community pharmacies, they were in favour of the development of extended professional services. ${ }^{17}$ S1BQ2f assesses the consumer's perception on the overall attitude, capability and service quality of the pharmacist and the pharmacy's staff. $31 \%$ of consumers were of the opinion that the respective attributes assessed were very good, 57\% of consumers responded fairly good, 9\% reported fairly poor, 3\% responded 'very poor' to the question. S1BQ3a assesses consumers' perception on pharmacist's ability to provide advice on current health problems. Those aged 16 to 19 years though that the pharmacist did fairly well; $4 \%$ of consumers opined that the pharmacist was not able to properly advise them on current health problems. S1BQ3c assesses consumers' perception on pharmacist's ability to handle medication disposing; $42 \%$ felt that the pharmacist did a fairly well job in disposing of medicines that were no longer needed.

The responses were for each item under the PSQ-3 domain were deemed statistically significant, implying that the majority of the respondents felt or perceived the same way (moderately satisfied; average score 77.14) regarding their perception of the community pharmacy they mostly went to and the pharmaceutical care services offered in those establishments. The PSQ-18 though a modified, concise version of the Patients' Satisfaction Questionnaire-3, revealed an average score of 45.60 ('poor' grade in terms of satisfaction). A plausible reason behind this finding can be found in earlier studies which conclusively stated that measuring patients' (customers/consumers) satisfaction must integrate dimensions of social, interpersonal, technical and moral facets of pharmaceutical care. ${ }^{18}$ Patient satisfaction with healthcare services in advanced and developing countries has many common and some unique variables and attributes that influence overall patient satisfaction. ${ }^{19}$

The average scores for PSQ-3 (77.14) and PSQ-18 (45.60) are different in terms of the grades. Though the PSQ-18 is a derivative of PSQ-3, it is presumed that the mean score and the corresponding score grades would be similar. However, that is not the case in this context. The explanation for these findings could be due to individual variability and the convenience sampling technique.

All the three domains in the current study (CPPQ, PSQ-3 and PSQ-18) assessed the same concept, which was consumers' satisfaction with the pharmaceutical services. The researchers wanted to establish if there was any degree of agreement between the three domains. For this purpose, the Kappa concordant test was utilized.

The Kappa concordant test performed for the three pairs (set 1 vs set 2; set 1 vs set 3 ; and set 2 vs set 3 ) revealed that there was no degree of agreement between the pairs. This observation was drawn based on the negative scores for measure of agreement between the three sets. ${ }^{13}$ The correlation and degree of agreement between the three domains was performed using Spearman's correlational test, the main purpose being that all three domains (CPPQ, PSQ-3 and PSQ-18) were standard validated questionnaires assessing the same concept, customers' satisfaction with the community pharmacy services provided. The three questionnaires also have the similar scoring patterns. ${ }^{11,20-23}$ The research investigated whether there was any chance of agreement between them for the purposes of adapting and/or adopting these three instruments for any research which would assess the satisfaction levels of the customers with regard to the community pharmacy services provided. However, the test revealed that there was no correlation between any of the sets. The inference with regard to this data is that though these questionnaires assess the same concept, each of them are 'stand-alone' entities with regard to patient satisfaction with community pharmacy services. An aspiring researcher cannot combine all these three questionnaires to create a separate questionnaire assessing customers' satisfaction with community pharmacy services.

Pharmacy education today emphasizes the provision of patient-orientated services. Patient interaction is a very important key element in a pharmacist's role. The consumers' perception of the benefits of pharmaceutical care is based on the ability of the pharmacist to help them. There might be different expectations from the patients as compared to the pharmacist's perspective regarding the role that pharmacist and their services. This will in return influence how patients perceive about community pharmacy regarding dispensing prescription and non-prescription 
drugs, medication advices and supplements or medical devices, health promotion campaigns and the capability of pharmacists. ${ }^{20}$

\section{Study Limitations}

A larger clustered sample would provide more clear data. A team of data collectors aiding the principal investigator can make the logistical process less cumbersome, provided appropriate training is conducted for the data collectors.

\section{CONCLUSION}

The current study utilized three study instruments which assessed customers' responses regarding community pharmacy services, to investigate the degree of correlation and agreement between them. The results analyses revealed that there did not exist any degree of agreement between the three instruments, despite the similarities. Fortifying this observation was the score grades assessment, wherein all the three sets, especially the PSQ-3 and PSQ-18 gave extremely varied scores and corresponding score grades. Though the three instruments measured and analyzed the same concept, each of them is distinctive. For future research involving patients' satisfaction with community pharmacy services, the aspiring researcher(s) must exercise extreme caution while combining these three questionnaires to create an adapted version of a new questionnaire to assess the same concept.

\section{ACKNOWLEDGEMENT}

The authors wish to acknowledge Faculty of Pharmacy (AIMST University) for enabling us to conduct this research.

\section{CONFLICT OF INTEREST}

The authors declare that there are no conflicts of interest.

\section{ABBREVIATIONS}

CPPQ: Community Pharmacy Patient Questionnaire; ICF: Informed Consent Form; IPF: International Pharmaceutical Federation; MEs: Medication Errors; PSQ-3: Patient Satisfaction Questionnaire-3; PSQ18: Patient Satisfaction Questionnaire-18; QoL: Quality of Life; WHO: World Health Organization.

\section{REFERENCES}

1. Shapiro AK, Shapiro E. The powerful placebo: From ancient priest to modern physician. JHU Press. 2000
2. Worley MM, Schommer JC, Brown LM, Hadsall RS, Ranelli PL, Stratton TP, et al. Pharmacists' and patients' roles in the pharmacist-patient relationship: Are pharmacists and patients reading from the same relationship script?. Res Social Adm Pharm. 2007;3(1):47-69.

3. Larson LN, Rovers JP, MacKeigan LD. Patient satisfaction with pharmaceutical care: Update of a validated instrument. J Am Pharm Assoc. 2002;42(1):44-50.

4. Cleary PD, McNeil BJ. Patient satisfaction as an indicator of quality care. Inquiry. 1988;25(1):25-36.

5. Haji MSE, Salem S, Mansoor H. Public's attitudes towards community pharmacy in Qatar: A pilot study. Patient Prefer Adherence. 2011;5:405.

6. Kenny K, Madhavan P. Dispensary separation: Perceptions of the public visiting primary care clinics in Malaysia. Malays J Pharm Sci. 2018;16:23-5.

7. Nahata MC. Challenges Facing Pharmacy Practice. Am Pharm. 1990;30(1):17-9.

8. Thamby SA, Subramani P. Seven-star pharmacist concept of WHO. J Young Pharm. 2014;6(2):1-3

9. McCrum-Gardner E. Sample size and power calculations made simple. Int J Ther Rehabil. 2010;17(1):10-4.

10. Community Pharmacy Patient Questionnaire (CPPQ). Available from: https:// psnc.org.uk/contract-it/essential-service-clinical-governance/cppq/. Retrieved on 19 February 2018.

11. Patient Satisfaction Questionnaire. Available from: https://www.rand.org/ health/surveys_tools/psq.html. Retrieved on 12 February 2018

12. John J. The knowledge, attitude, practice and perceived barriers towards screening for premalignant cervical lesions among women aged 18 years and above, in Songea urban, Ruvuma: (Doctoral dissertation, Muhimbili University of Health and Allied Sciences). 2011.

13. Chmura KH, Periyakoil VS, Noda A. Kappa coefficients in medical research. Stat Med. 2002;21(14):2109-29.

14. Emerson RW. Convenience sampling, random sampling and snowbal sampling: How does sampling affect the validity of research?. J Vis Impair Blind. 2015;109(2):164-8.

15. Boardman $H$, Lewis M, Croft $P$, Trinder $P$, Rajaratnam G. Use of community pharmacies: A Population-based survey. J Public Health. 2005;27(3):254-62.

16. Al-Arifi MN. Patients' perception, views and satisfaction with pharmacists' role as health care provider in community pharmacy setting at Riyadh, Saudi Arabia. Saudi Pharm J. 2012;20(4):323-30

17. Wirth F Tabone F, Azzopardi LM, Gauci M, Zarb-Adami M, Serracino-Inglott A. Consumer perception of the community pharmacist and community pharmacy services in Malta. J Pharm Health Serv Res. 2010;1 (4):189-94.

18. Marley KA, Collier DA, Meyer GS. The role of clinical and process quality in achieving patient satisfaction in hospitals. Decis Sci. 2004;35(3):349-69.

19. Ahmad I, Nawaz A, Khan S, Khan H, Rashid MA, Khan MH. Predictors of patient satisfaction. Gomal J Med Sci. 2011;9(2):183-8.

20. Panvelkar PN, Saini B, Armour C. Measurement of patient satisfaction with community pharmacy services: A review. Pharm World Sci. 2009;31(5):525-37.

21. Marshall GN, Hays RD. The patient satisfaction questionnaire short-form (PSQ-18). 1994

22. Thayaparan AJ, Mahdi EJ. The Patient Satisfaction Questionnaire Short Form (PSQ-18) as an adaptable, reliable and validated tool for use in various settings. Med Educ Online. 2013;18.

23. World Health Organization. The role of the pharmacist in the health care system Good pharmacy practice. Geneva: World Health Organization. 1994.

Article History: Submission Date : 13-06-2019; Revised Date : 13-07-2019; Acceptance Date : 21-08-2019.

Cite this article: How CLT, Thamby SA, Ping NY. Evaluation of a Study Instrument Assessing Community Pharmacies and Services Provided: Consumers' Perception. J Young Pharm. 2019;11(4):386-90 